\title{
MATTER DISTRIBUTION IN THE GALAXY CLUSTERS A 539
} AND A 2319

\author{
D. TRÈVESE, G. CIRIMELE AND M. DE SIMONE \\ Istituto Astronomico, Università di Roma "La Sapienza" \\ Via G.M. Lancisi 29, Roma I-00161
}

We performed a combined X-ray and optical analysis of the two clusters A539 and A2319, based on ROSAT PSPC 0.4-2.4 keV images of the public archive and $\mathrm{F}$ band photometry from microdensitometric scans of Palomar 48 inch plates (Trèvese et al. 1992, A\&AS, 94, 327). Assuming spherical symmetry and following the methods adopted in Cirimele, Nesci, and Trèvese (1997, ApJ, 475, 11 (CNT97)) we derived the radial distribution of gas and galaxy densities $\rho_{\text {gas }}$ and $\rho_{\text {gal }}$ and we have computed the morphological parameter $\beta_{x o} \equiv d \ln \rho_{\text {gas }}(r) / d \ln \rho_{\text {gal }}(r)$, introduced in CNT97. This allows to check the validity of the hydrostatic equilibrium condition, which $\operatorname{reads} \beta_{s p e c}(r) \equiv \frac{\mu m_{p} \sigma_{r}^{2}}{k T(r)}=\beta_{x o}(r)+d \ln T / d \ln \rho_{g a l}$, for an isotropic and uniform velocity distribution of r.m.s. dispersion $\sigma_{r}$. In the case of A539, adopting $\sigma_{r}=629 \mathrm{~km} \mathrm{~s}^{-1}$ from Fadda et al. (1996, ApJ, 473,670 ) and $\mathrm{T}=1.57 \mathrm{keV}$ David et al. (1996, ApJ, 473, 692), we obtained marginally consistent values of $\beta_{\text {spec }}=1.54 \pm 0.50$ and $\beta_{x o}=1.08 \pm 0.11$. In the case of A2319 we took into account the presence of the secondary component A2319B (Oegerle et al. 1995, AJ, 110, 32) and the temperature gradient (Markevitch M. 1996, ApJ, 465, L1). The resulting radial increase of $\beta_{\text {spec }}$ is consistent with that of $\left(\beta_{x o}(r)+d \ln T(r) / d \ln \rho_{g a l}\right)$, suggesting that the hydrostatic equilibrium holds also in the presence of a temperature gradient. The radial distribution of the total binding mass, the mass in galaxies and intergalactic gas show that in both clusters the gas mass profile is steeper than galaxies and total masses consistently with our previous results (CNT97). Adopting a constant gas temperature, the relevant baryon fractions are larger than $20 \%$, adding new evidence to the "baryon catastrophe". Taking into account the radial decrease of gas temperature, the baryon fraction is further increased. This implies that either $\Omega_{o}<0.25$, or that large halos of dark matter surround galaxy clusters, as suggested by White \& Fabian (1995, MNRAS, 273, 72). 